\title{
Afferent Drive of Medial Prefrontal Cortex by Hippocampus and Amygdala is Altered in MAM-Treated Rats: Evidence for Interneuron Dysfunction
}

\author{
Behnaz Esmaeili*,1,2,3 and Anthony A Grace ${ }^{1,2,3}$ \\ 'Department of Neuroscience, University of Pittsburgh, Pittsburgh, PA, USA; ${ }^{2}$ Department of Psychiatry, University of Pittsburgh, \\ Pittsburgh, PA, USA; ${ }^{3}$ Department of Psychology, University of Pittsburgh, Pittsburgh, PA, USA
}

\begin{abstract}
Evidence indicates that the prefrontal cortex and its regulation by afferent inputs are disrupted in schizophrenia. Using a validated rat model of schizophrenia based on prenatal administration of the mitotoxin methyl azoxymethanol acetate (MAM), we examined the convergent projections from the ventral hippocampus (vHipp) and the basolateral amygdala (BLA) in the medial prefrontal cortex (mPFC). In vivo extracellular recordings were done in anesthetized rats to assess how prior stimulation of the BLA or vHipp input to the mPFC affected mPFC responses to subsequent stimulation of these regions. The interstimulus interval (ISI) of the BLA and vHipp pulse stimulation was varied randomly between 0 and $130 \mathrm{~ms}$, and the probability of evoked spike response in the mPFC measured. We found that BLA input increased vHipp-evoked spike probability at ISIs 40-130 ms, but decreased spike probability at ISIs I0-20 ms. This would be consistent with activation of inhibitory interneurons at shorter ISIs by BLA stimulation. In contrast, in MAM-treated rats BLA stimulation increased vHipp-evoked spike probability in mPFC at all ISIs tested. Given that interneurons are driven primarily by N-methylD-aspartate (NMDA) channel activation, the effects of the NMDA channel blocker, phencyclidine (PCP), were tested. PCP was found to completely attenuate the inhibitory effect of BLA input on vHipp-evoked responses in MPFC at shorter ISIs, causing the response in control rats treated with PCP to resemble that observed in the MAM rat. In contrast to the effects of BLA stimulation on vHipp-mPFCevoked responses, there was no inhibitory period when examining the effects of $v$ Hipp stimulation on BLA-mPFC-evoked responses in control rats, but in MAM-treated rats there was a significant inhibition at short intervals. Thus, both affective input arising from the BLA and context-dependent input from the vHipp exert a modulatory effect on mPFC neural activity in response to these inputs. Whereas the BLA potentiated $\mathrm{vH}$ Hipp input to the $\mathrm{mPFC}$ at long intervals, there was a short-interval inhibitory period that appeared to be mediated by an NMDA-dependent drive of interneurons. This inhibitory modulation was absent in the model of schizophrenia and following PCP, which is consistent with an interneuron disruption in this disorder.
\end{abstract}

Neuropsychopharmacology (2013) 38, I87I-1880; doi:I0.1038/npp.20I3.64; published online 27 March 2013

Keywords: amygdala; hippocampus; prefrontal cortex; NMDA; schizophrenia

\section{INTRODUCTION}

The medial prefrontal cortex (mPFC) receives converging inputs from the ventral hippocampus (vHipp) (Floresco and Grace, 2003; Ishikawa and Nakamura, 2003), a region related to context-dependent processing, (Corcoran et al, 2005; Ji and Maren, 2007; Phillips and LeDoux, 1992; Selden et al, 1991) and the basolateral amygdala (BLA), which provides an important affect-related input to the MPFC, and that has a prominent role for the processing and integration of emotionally salient sensory information and learning

\footnotetext{
*Correspondence: Dr B Esmaeili, Department of Neuroscience, University of Pittsburgh, A210 Langley Hall, University of Pittsburgh, Pittsburgh, PA 15260, USA, Tel: + 412624 7332, Fax: + | 412624 9198, E-mail: behnaz.esmaeili@gmail.com

Received 22 December 2012; revised 27 February 2013; accepted 4 March 2013; accepted article preview online 7 March 2013
}

(Garcia et al, 1999; Laviolette et al, 2005; Milad and Quirk, 2002; Milad et al, 2004; Rosenkranz and Grace, 2002). Together, these systems have a role in the regulation of fear memory, reward associated learning, drug addiction, and anxiety disorders. In the rat, the vHipp has glutamatergic projections to both pyramidal neurons and GABAergic interneurons of the prelimbic region of the MPFC (Carr and Sesack, 1996; Gabbott et al, 2002, 2006). Stimulation of the hippocampus is known to evoke excitatory and inhibitory responses in mPFC neurons (Gigg et al, 1994; Jay et al, 1995). The terminals of the BLA projections are found in close proximity to vHipp terminals on PFC neurons (Bacon et al, 1996; Gabbott, 2003). Extracellular in vivo recordings have shown that excitatory and evoked inhibitory inputs driven by BLA and vHipp stimulation converge and interact in the medial and orbital PFC (Ishikawa and Nakamura, 2003). Paired activation of BLA/vHipp afferents can exert two differential effects on the evoked activity of 
PFC neurons: (1) an inhibition of firing evoked by lowfrequency stimulation of the BLA/vHipp at short latencies and (2) an enhancement of mPFC evoked firing at long latencies (Ishikawa and Nakamura, 2003). The above mentioned findings suggest that converging cortical inputs originating from the BLA and the hippocampus may interact to regulate cognitive functions governed by the mPFC. Despite this, there is a paucity of research investigating the mechanisms by which these inputs interact to influence mPFC neural activity.

Evidence indicate that the prefrontal cortex and its regulation by afferent inputs are disrupted in schizophrenia (Goldberg and Weinberger, 1988; Lewis and Moghaddam, 2006; Lewis, 2012). Impaired functional coupling between the $\mathrm{mPFC}$ and hippocampus is proposed to contribute to the impaired cognition and emotional regulation in schizophrenia patients (Allen et al, 2012; Wolf et al, 2009; Zhou et al, 2008). In addition, the amygdala, which may have a role in schizophrenia (Benes, 2010), potently interacts with the mPFC. Prenatal administration of methylazoxymethanol acetate (MAM), an antimitotic agent, leads to anatomical and behavioral disruptions in adult rodents that are comparable to some of the deficits that have been described in schizophrenia patients (Grace and Moore, 1998; Flagstad et al, 2004; Gourevitch et al, 2004; Moore et al, 2006). On the basis of this model, we have previously suggested a decrease in intrinsic GABAergic signaling may be responsible for the prefrontal and hippocampal dysfunction observed during task performance and in evoked gamma band activity (Lodge et al, 2009), which is consistently observed in animal models as well as in schizophrenia in humans (Cunningham et al, 2006; Lewis and Hashimoto, 2007; Lewis et al, 2012). Furthermore, the density of parvalbumin (PV)-positive interneurons throughout the mPFC and ventral subiculum of the hippocampus is known to be decreased in MAM model, as also observed in schizophrenia brains postmortem (Lewis et al, 2005; Lodge et al, 2009; Penschuck et al, 2006; Woo et al, 1998). These PV interneurons are known to be driven primarily by $N$-methyl-D-aspartate (NMDA) receptors (Behrens et al, 2007; Bitanihirwe et al, 2009; Whittington et al, 1995). Indeed, the NMDA hypofunction model of schizophrenia (Coyle, 1996; Javitt and Zukin, 1991; Jodo et al, 2003) is based in part on a diminished NMDA drive of interneurons, resulting in prefrontal hyperactivity and cognitive impairment (Lewis and Moghaddam, 2006; Lewis, 2012). Furthermore, administration of NMDA receptor antagonists such as phencyclidine (PCP) induces cortical excitation and transient schizophrenia-like symptoms in human and non-human animals (Cohen et al, 1962; Domino and Luby, 2012; Moghaddam and Adams, 1998).

In this study, we examined how stimulation of the BLA or vHipp affects subsequent vHipp or BLA afferent input to the $\mathrm{mPFC}$, using in vivo extracellular single-unit recordings. Given the potential relevance of this network to schizophrenia, the evidence of a GABA dysfunction in schizophrenia patients, and an association between GABA interneurons and coordinated neuronal activity, the effect of the BLA- or vHipp-evoked response in mPFC on the alternate input was tested in the MAM model of schizophrenia. These results were compared with a pharmacological model of frontal cortical dysfunction in schizophrenia by administering the NMDA channel blocker PCP.

\section{MATERIALS AND METHODS}

\section{Subjects and Materials}

Electrophysiological recordings were conducted in male Sprague-Dawley rats and male offspring of MAM- and saline-treated pregnant female rats (Hilltop, Scottsdale, PA). All experiments and drug treatments performed in this study were in accordance with the guidelines outlined in the United States Public Health Service Guide for the Care and Use of Laboratory Animals, and were approved by the Institutional Animal Care and Use Committee of the University of Pittsburgh. Adult male rats weighing $300-400 \mathrm{~g}$ arrived at least 1 week before use and were housed two per cage in a temperature $\left(22^{\circ} \mathrm{C}\right)$ and humidity (47\%)-controlled environment on a 12 -h light/dark cycle (light on at 07:00 hour); food and water were available ad libitum. Timed pregnant female Sprague-Dawley dams (Hilltop) arrived at GD15, and were housed individually on a normal 12-h light cycle (lights on at 07:00 hour). At GD17, pregnant females were randomly separated into two groups; one group received injection of $0.9 \%$ saline (i.p., $1 \mathrm{ml} / \mathrm{kg}$ ), whereas the other group was injected with $20 \mathrm{mg} / \mathrm{kg}$ MAM (Midwest Research Institute) in saline, as previously described (Moore et al, 2006). Within 3 weeks from birth, male offsprings were weaned and housed in pairs or groups of three with littermates.

The NMDA receptor channel blocker PCP hydrochloride was obtained from Sigma-Aldrich, and initially dissolved in $0.9 \%$ saline for i.v. injection. The dose of PCP used in the present study $(0.4 \mathrm{mg} / \mathrm{kg})$ was chosen based upon prior reports (Santana et al, 2011) and our pilot study.

\section{Surgery}

Rats were anesthetized with an initial dose of chloral hydrate $(400 \mathrm{mg} / \mathrm{kg}$, i.p.), and anesthesia was maintained by periodic supplemental injection (i.p.) to maintain suppression of the hindlimb withdrawal reflex, tested approximately every $30 \mathrm{~min}$. Rats were placed in a stereotaxic frame (Kopf) and then, in some experiments, implanted with a catheter in the lateral vein of the tail to allow i.v. injections. Body temperature was maintained at $37^{\circ} \mathrm{C}$ using a temperature-controlled heating pad (Fintronics).

\section{Single-Unit Extracellular Recording in mPFC}

Single-glass microelectrodes (WPI; impedance 7-10 M 2 ) were pulled on a Narishige PE-2 vertical electrode puller, broken back under microscopic control, and filled with $2 \%$ Pontamine sky blue dye in $2 \mathrm{M} \mathrm{NaCl}$; final impedance was 8-12 M $\Omega$. Electrodes were placed using a hydraulic microdrive (Narishige) into the $\mathrm{mPFC}$, according to coordinates in a rat brain atlas (Paxinos and Watson, 1986; anteroposterior (AP) $2.5-3.2 \mathrm{~mm}$ anterior to bregma; lateral (L), $0.6-0.8 \mathrm{~mm}$ to the midline; dorsoventral (DV), $2.5-4.5 \mathrm{~mm}$ from the top of the dura). Neural activity was amplified and filtered (500-5000 Hz, Fintronics) before transferring to a computer with custom-designed acquisition and analysis software 
(Neuroscope; Brian Lowry, Pittsburgh, PA). Spontaneous activity was monitored on an oscilloscope and single-unit responses were recorded when the signal to noise ratio was at least 3:1.

\section{Stimulation of BLA and vHipp}

Concentric bipolar stimulating electrodes (NEX-100X; Rhodes Medical Instruments) were placed in the BLA (AP, -3.6 posterior to bregma; $\mathrm{L}, 4.8 \mathrm{~mm}$ from the midline; $\mathrm{D}, 8.2 \mathrm{~mm}$ from the dura) and vHipp (AP, -6 posterior to bregma; $\mathrm{L}, 4.5 \mathrm{~mm}$ from the midline; $\mathrm{D}, 7.3 \mathrm{~mm}$ from the dura). Single-pulse stimulation (duration, $0.25 \mathrm{~ms}$; intensity, $1 \mathrm{~mA}$ ) was applied alternately to the BLA and vHipp (130 ms intervals, every $2 \mathrm{~s}$ ), as the recording electrode was slowly advanced in the mPFC. Single-current pulses were generated using a dual-output stimulator (S8800; Grass Technologies). Neurons in mPFC were recorded only if they were orthodromically responsive to both inputs from the vHipp and BLA. Cells that did not respond monosynaptically to the BLA stimulation (mean spike latency, <22 ms; (Laviolette et al, 2005)) and/or vHipp stimulation (mean spike latency, <22 ms; (Ishikawa and Nakamura, 2003)) were not used for data analysis. Upon identifying a neuron in $\mathrm{mPFC}$ responding to both vHipp and BLA input with monosynaptic and orthodromic responses, stimulation currents were adjusted to threshold or subthreshold level stimulation intensity (range $0.1-1.2 \mathrm{~mA}$ ) so that stimulation of the vHipp or BLA would evoke an action potential of $\sim 50 \%$ (range $40-60 \%$ ) or $1-4 \%$ of the time, respectively, in response to the single-pulse stimulation delivered at $0.5 \mathrm{~Hz}$. We used various stimulation protocols designed to investigate different types of interactions between hippocampal and BLA inputs to the MPFC.

Interactions between Converging Inputs from the vHipp and BLA in the MPFC

A series of paired-pulse stimulation experiments were conducted to assess how stimulation of one input could influence the response evoked by stimulation of the second input. This was performed in MPFC neurons that displayed a monosynaptic orthodromic spike after stimulation of both the BLA and vHipp. Each neuron in mPFC received a pulse stimulation of one input followed by a second pulse of the other input. Basal vHipp- or BLA-evoked activity was recorded for 3-5 min at a threshold level (50\% spike probability), and then the alternate input (BLA or vHipp, respectively) was added at a subthreshold level of stimulation (1-4\% spike probability). The interstimulus interval (ISI) of the BLA/vHipp pulse pair was then varied between 0 and $130 \mathrm{~ms}$.

The effect of the subthreshold stimulation of the BLA or vHipp on the vHipp- or BLA-evoked firing, respectively, was also assessed in MPFC in MAM and saline control rats. Stereotaxic coordinates for MAM rats were adjusted on the basis of the previous works of our group (Goto and Grace, 2006; Lodge et al, 2009), and from results of a pilot study as follows: BLA (AP, -3.6 posterior to bregma; $\mathrm{L}, 4.8 \mathrm{~mm}$ from the midline; $\mathrm{D}, 8 \mathrm{~mm}$ from the dura); vHipp (AP, -6 posterior to bregma; $\mathrm{L}, 4.8 \mathrm{~mm}$ from the midline; $\mathrm{D}, 6.9 \mathrm{~mm}$ from the dura); and mPFC (AP, 2.5-3 anterior to bregma; $\mathrm{L}$, $0.5-0.7 \mathrm{~mm}$ from the midline; $\mathrm{D}, 2.5-3.6 \mathrm{~mm}$ from the dura).

\section{Interactions between vHipp and BLA Inputs in the mPFC After NMDA Channel Blockade}

In control rats, after identifying a neuron in mPFC responding to both vHipp and BLA input, basal vHippevoked activity was recorded for $3 \mathrm{~min}$ and then BLA input was added at ISIs $130,100,80,40,20$, and $10 \mathrm{~ms}$, in a random arrangement (50-100 trials/ISI). Following this basal recording, rats were administered PCP $(0.4 \mathrm{mg} / \mathrm{kg}$, i.v.) and vHipp-evoked responses and the effect of BLA stimulation on vHipp-evoked response was recorded at the same ISIs as before PCP administration.

\section{Histology}

At the end of each experiment, the final position of the recording electrode was marked by an ionotophoretic ejection of Pontamine Sky Blue dye from the tip of the electrode (Kation Scientific; $-25 \mu \mathrm{A}$ constant current for $40 \mathrm{~min})$. The stimulation electrode placements were marked by electrolytic lesion ( $100 \mu \mathrm{A}$ for 10 -s current pulse) and visualized by adding potassium ferrocyanide during postfixation. The rats were euthanized by an overdose of chloral hydrate (additional $400 \mathrm{mg} / \mathrm{kg}$, i.p.) and decapitated. The brains were removed and fixed for at least $48 \mathrm{~h}(8 \% \mathrm{w} / \mathrm{v}$ paraformaldehyde in PBS), and cryoprotected $(25 \% \mathrm{w} / \mathrm{v}$ sucrose in PBS) until saturated. Brains were sectioned $(60-\mu \mathrm{m}$ coronal sections), placed on gelatin-chromealum-coated slides, and stained with cresyl violet for histochemical verification of the recording/stimulation sites.

\section{Data Analysis}

Data for evoked spike probabilities were calculated by dividing the number of stimuli that evoked an action potential by the total number of stimuli applied. Data were assessed by one-way repeated measures ANOVA, with time as the within-subject factor. Multiple comparisons were analyzed using a two-way ANOVA, with treatment as the between-subject factor and time as the within-subject factor. Where necessary, post hoc analysis was carried out with Dunnet's or Tukey's multiple comparison tests to determine the changes in spike probability. Significance was set at $p<0.05$. Calculations were performed using SPSS statistical software package (version 11.5).

\section{RESULTS}

\section{BLA- and vHipp-Evoked Spiking in mPFC}

Extracellular activity was recorded from both prelimbic and infralimbic segments of the mPFC neurons in chloral hydrate-anesthetized rats (Figure 1a). There were no significant differences between prelimbic and infralimbic neuronal responses, so responses from both segments were pooled. Stimulation electrodes were placed in the vHipp (Figure 1b) and in the BLA (Figure 1c). Medial prefrontal cortical (mPFC) neurons have been shown to receive converging inputs from the vHipp and the BLA (Ishikawa 


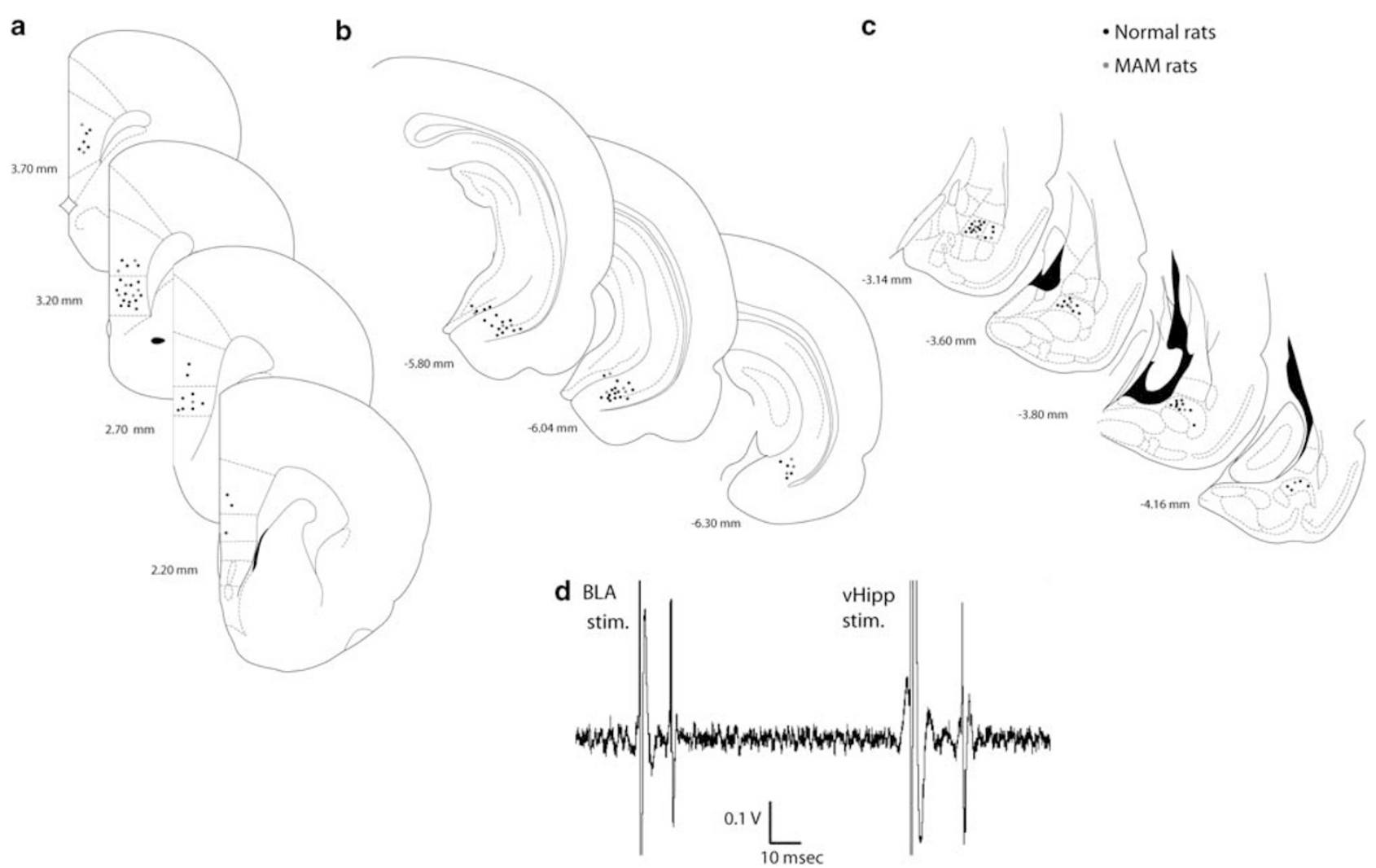

Figure I (a-c) Schematic of coronal sections of the rat brain showing representative placements (circles, for clarity, 50\%) of recording electrodes in the mPFC (a), and stimulating electrodes in the vHipp (b) and the BLA (c). The brain sections correspond to the atlas of Paxinos and Watson (1986). The numbers correspond to millimeters from bregma. (d) mPFC neuron excited by BLA and vHipp, following stimulation of these regions.

and Nakamura, 2003). Single-pulse stimulation of these afferent areas evoked action potential firing in $40-60 \%$ of mPFC neurons. mPFC neurons were selected that received excitatory input from both vHipp and BLA, which was indicated by orthodromically evoked spikes, following stimulation of these structures (Figure 1d), with putative orthodromic activation defined by variable latency-evoked responses. The mean evoked spike latency of the monosynaptic and orthodromic response of mPFC neurons responding to vHipp $(n=42)$ or BLA $(n=9)$ stimulation was $18.1 \pm 0.45 \mathrm{~ms}$ and $17.8 \pm 1.43 \mathrm{~ms}$, respectively, which is consistent with previous reports (Ishikawa and Nakamura, 2003; Laviolette et al, 2005). The mean baseline spike probability was $35-65 \%$, and the baseline current intensity to obtain $50 \%$ spike probability was $200-1200 \mu \mathrm{A}$.

\section{BLA Input Effect on vHipp-Evoked Firing in mPFC}

In $\mathrm{mPFC}$ neurons that displayed a monosynaptic spike in response to stimulation of both the vHipp and the BLA, a sequential-pulse stimulation protocol was used to evaluate how stimulation of one input could influence firing driven by the other input. The vHipp was stimulated at threshold current intensities (50\% evoked spike probability), and the BLA was stimulated at subthreshold current (1-4\% evoked spiking) before the vHipp pulse at 130-, 100-, 80-, 40-, 20-, $10-$, and 0 -ms intervals. BLA input resulted in significant changes in vHipp-evoked spike probability at ISIs $0-130 \mathrm{~ms}$ $(n=42$, one-way ANOVA; $\mathrm{F}(7,259)=54.804 ; p<0.001)$ compared with the vHipp stimulation alone (Figure 2a). Results showed that subthreshold stimulation of the BLA increased mean spike probability at ISIs $130,100,80$, and $40 \mathrm{~ms}$ by $30-70 \%$ (mean $59.2 \pm 19.3 ; p<0.05$ ); the excitation of vHipp-evoked firing probability was maximal when the BLA stimulation pulse was administered $100 \mathrm{~ms}$ before vHipp stimulation $(p<0.001$; Dunnett's post hoc test), and was the lowest at ISI of $40 \mathrm{~ms}(30 \% ; p<0.05)$. In contrast, BLA single-pulse stimulation at shorter intervals (ie, 20 and $10 \mathrm{~ms}$ ) decreased baseline vHipp-evoked firing in mPFC between $60 \%$ and $80 \%$, respectively (mean $70 \pm 14.1$; $p<0.001)$. BLA and vHipp stimulation were delivered at the same time $(\mathrm{ISI}=0)$ for $18 \mathrm{mPFC}$ neurons responsive to both stimulations; $12 / 18$ neurons showed an inhibition of firing compared with the threshold level of vHipp-evoked activity $(\mathrm{t}(11)=-13.1, p<0.001)$ and $6 / 18$ neurons responded at the same level as or higher than the basal vHipp-evoked activity in $\operatorname{mPFC}(\mathrm{t}(5)=3.5, p<0.05)$.

\section{vHipp Input Effect on BLA-Evoked Response in mPFC}

The symmetry of the BLA/vHipp interaction was tested in nine additional mPFC neurons, in which subthreshold vHipp pulses were paired with threshold (50\%) stimulation of BLA (vHipp before BLA). For these experiments, only interpulse intervals of $130,80,40,20,10$, and $0 \mathrm{~ms}$ (Figure 2b) were used because we did not observe any significant difference between ISIs 80-, 100-, and 130-ms intervals, and the magnitude of excitation of firing was 


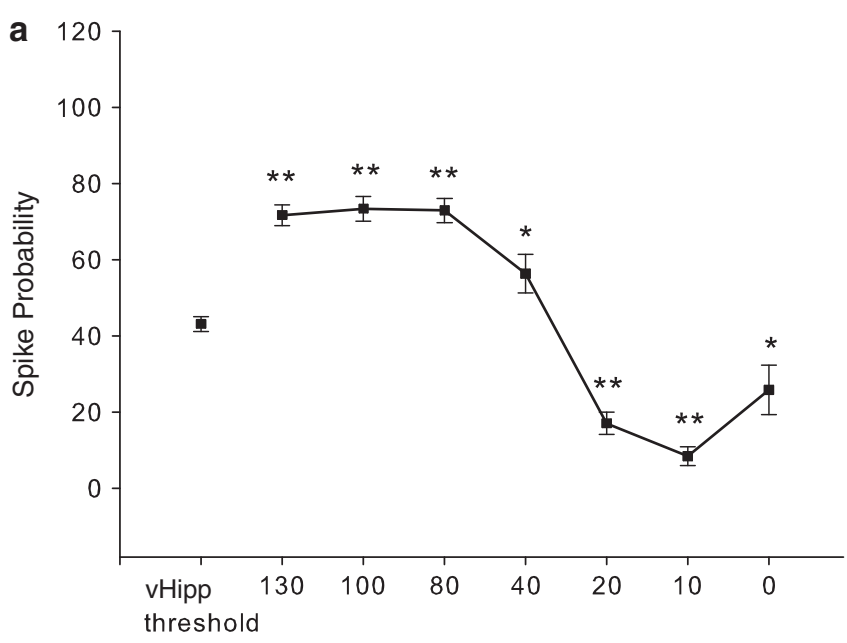

Pre-vHipp stimulation (msec)

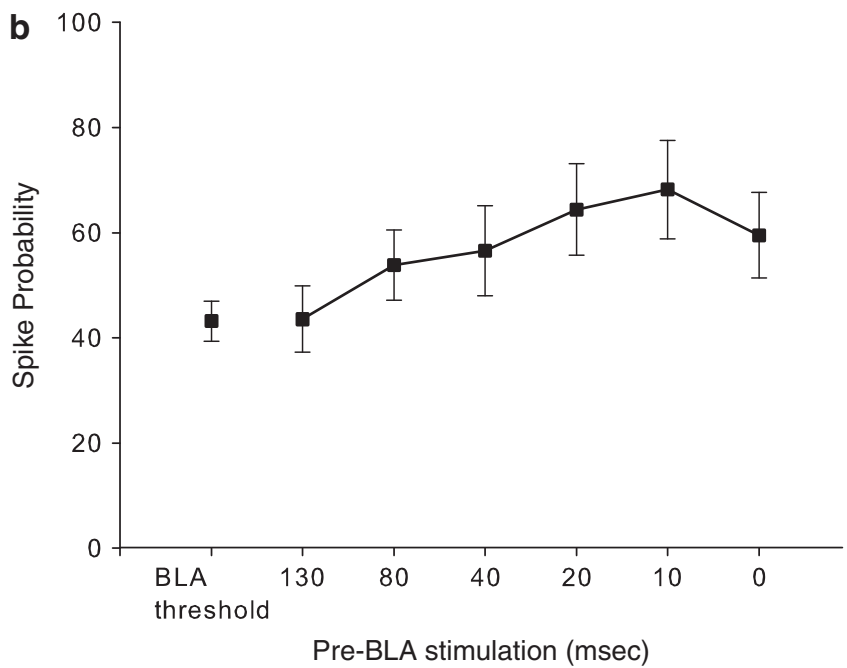

Figure 2 Effect of the BLA or $v$ Hipp input to the mPFC on subsequent stimulation of these regions. (a) Subthreshold BLA stimulation was paired with threshold vHipp stimulation at different ISIs (BLA before vHipp as shown in Figure I). Subthreshold BLA stimulation increased vHipp-evoked spike probability at long ISIs, whereas at short ISIs there was an attenuation of response $(* * * 0.001$, $* p<0.05$ compared with vHipp-evoked SP at baseline; one-way ANOVA; Dunnett's post hoc test). (b) Subthreshold vHipp stimulation was paired with threshold BLA stimulation at different ISIs (vHipp before BLA). Subthreshold vHipp stimulation failed to significantly affect BLA-evoked spike probability across the ISIs tested.

nearly equivalent at intervals of 100 and $80 \mathrm{~ms}$. Application of single-pulse stimulation to the vHipp before a stimulation pulse to the BLA did not significantly affect the response to BLA stimulation (one-way ANOVA; $\mathrm{F}(6$, $61)=1.506 ; p>0.05)$. When threshold level of BLA and subthreshold level of the vHipp stimulation were applied together $(\mathrm{ISI}=0), \mathrm{mPFC}$ neurons responded at the same level as or higher than the threshold level of the BLA-evoked activity $(n=9)$. In contrast to the BLA/vHipp interaction where the BLA stimulation was applied before vHippevoked firing activity, shorter intervals of vHipp stimulation before the BLA (20 and $10 \mathrm{~ms}$ ) did not produce suppression of firing compared with the baseline BLA-evoked firing probability.

\section{BLA Input Effect on vHipp-Evoked Response in mPFC in MAM vs Saline Rats}

The above findings indicate that single-pulse stimulation of the BLA can exert a time-dependent modulation over mPFC neuron firing driven by inputs from the vHipp. Given the anatomy of the inputs, the inhibitory effect of the BLA stimulation at shorter ISIs is consistent with activation of inhibitory interneurons at shorter ISIs by BLA stimulation. Given that in MAM-treated rats and in other models of schizophrenia there is a reported decrease in PV-containing interneurons (Berretta et al, 2004; Lodge et al, 2009; Penschuck et al, 2006), we examined whether there was an alteration in the paired stimulation profile in the MPFC of MAM rats. In $14 \mathrm{mPFC}$ neurons from 14 MAM rats, and seven mPFC neurons from seven saline rats that also displayed monosynaptically evoked action potentials in response to vHipp and BLA stimulation, we assessed how single-pulse stimulation of the BLA modulates vHipp input into $\mathrm{mPFC}$ using this sequential-pulse stimulation protocol (Figure 3a). Analysis of these data revealed a significant ISI treatment interaction $(\mathrm{F}(7,125)=4.015 ; p<0.001)$ (Figure $3 \mathrm{a})$. In these neurons, the baseline firing probability evoked by vHipp stimulation in both MAM and saline treated rats did not differ (MAM baseline mean spike probability $=45.2 \pm 12.4$; saline mean spike probability $=$ $49.1 \pm 8.2 ; p>0.05$; Tukey's post hoc test). Moreover, subthreshold BLA stimulation increased vHipp-evoked spike probability at long ISIs (130-40) from 70 to $89 \%$ (mean $79.9 \pm 9.1$ ) in MAM groups as well as in saline groups (32.8-66.2\%; mean $45.6 \pm 16.1)$. The facilitation of vHippevoked spike probability was greatest at ISIs 40 and $100 \mathrm{~ms}$ in MAM and saline rats, respectively. In MAM-treated rats, subthreshold stimulation of the BLA increased evoked spike probability of vHipp drive of $\mathrm{mPFC}$ at shorter ISIs (ie, 10 and $20 \mathrm{~ms}$ ) from 31.5 to $45.4 \%$ (mean $38.4 \pm 9.8$ ), respectively, compared with the vHipp-evoked activity alone. Compared with the saline rats, the MAM rats failed to show the inhibition produced by BLA stimulation on the vHippevoked response in mPFC at short intervals $(p<0.001$; Tukey's post hoc test).

\section{vHipp Input Effect on BLA-Evoked Response in mPFC in MAM-Treated Rats}

Given evidence demonstrating the importance of the hippocampal-mPFC pathway in schizophrenia models and in human patients (Lodge et al, 2009; Zhou et al, 2008), we compared the effects of subthreshold activation of the vHipp on BLA-evoked firing in $\mathrm{mPFC}$ at ISIs $0-130 \mathrm{~ms}$ in MAM-treated rats (five neurons) with normal rats (nine neurons) (Figure 3b). A two-way ANOVA on the spike probability showed a significant difference between MAM and control animals $(\mathrm{F}(1,91)=5.210, p<0.05)$ (Figure $3 \mathrm{~b})$. MAM rats displayed a decreased spike probability at ISIs 20, 10 , and $0 \mathrm{~ms}$ compared with the normal rats; the difference between the BLA-evoked response at shorter ISIs (ie, 10 and $0 \mathrm{~ms}$ ) in MAM rats and that of normal rats was statistically significant (mean difference $=37.4-39.2 ; p<0.05$; Tukey's post hoc test). In contrast, at longer ISIs (ie, 130-20 ms) MAM rats did not show a significant difference in spike probability ( $p>0.05$; Tukey's post hoc test) compared with 

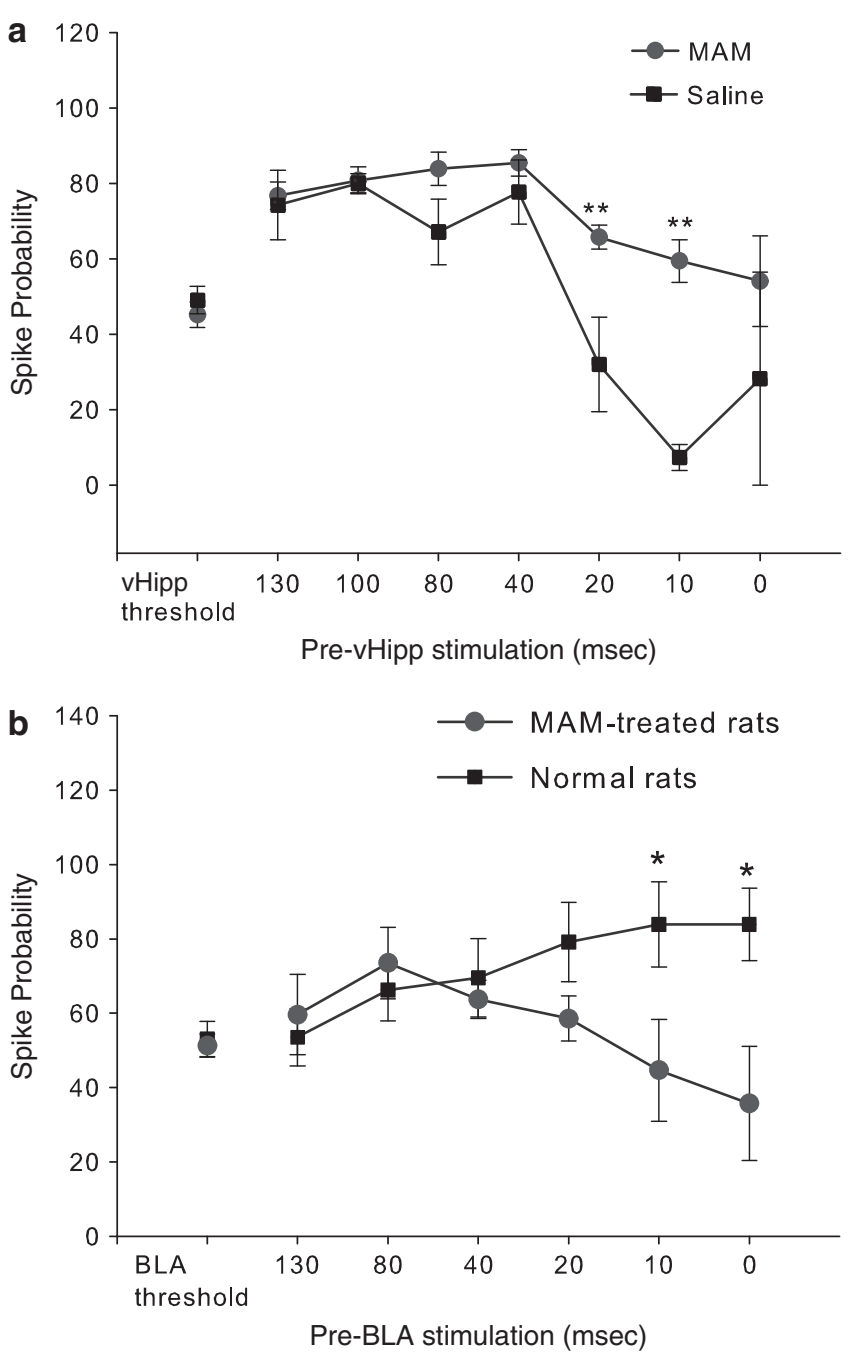

Figure 3 Interaction between the BLA and vHipp inputs to the mPFC in MAM- and saline-treated rats. (a) Effect of subthreshold BLA stimulation on vHipp-evoked spike probability in MPFC in MAM- and saline-treated rats. BLA input increased vHipp-evoked spike probability at long ISIs to a similar extent in both saline and MAM rats. In contrast, subthreshold BLA stimulation did not have any inhibitory effect on vHipp-evoked spike probability at short ISIs in MAM rats compared with saline rats. (** $p<0.00$ I, two-way ANOVA; Tukey's post hoc test). (b) Effect of subthreshold vHipp stimulation on BLA-evoked spike probability in mPFC in MAM- and saline-treated rats. Spike probability was normalized to BLA threshold in control group. In MAM-treated rats, vHipp stimulation showed a selective decrease in evoked spike probability at short ISIs compared with controls $(* 0<0.05)$

control rats. Data from the MAM-treated rats showed that the response to single-pulse stimulation of the vHipp before a stimulation pulse to the BLA was not significantly different compared with the BLA-evoked activity alone (one way ANOVA; $\mathrm{F}(6,29)=1.569 ; p>0.05$ ).

\section{NMDA Channel Blockade in Controls Mimicked the Response Observed in MAM Rats}

Given that interneurons are driven primarily by NMDA channel activation, we tested the effects of the indirect NMDA antagonist PCP on the interaction between BLA and vHipp stimulation on $\mathrm{mPFC}$-evoked responses. After

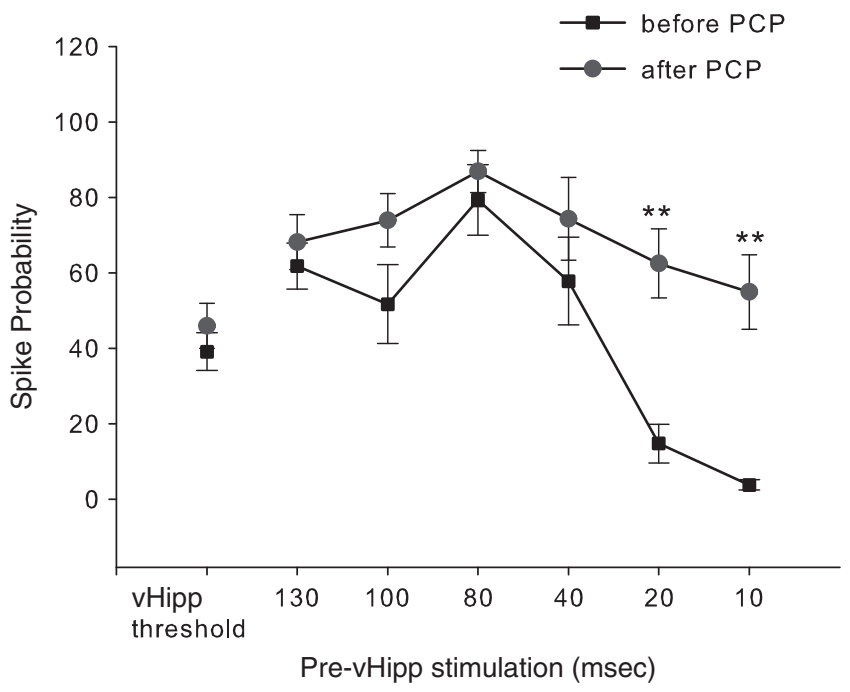

Figure 4 PCP blocks the inhibitory effect of BLA stimulation on vHippevoked activity. PCP administration to normal rats selectively attenuated BLA attenuation of VHipp drive of the MPFC at short ISIs. Administration of the NMDA channel blocker PCP $(0.4 \mathrm{mg} / \mathrm{kg}$, i.v. $)$ did not alter vHipp drive of mPFC neurons. PCP administration did not alter the BLA-mediated facilitation of $v$ Hipp-evoked spiking in MPFC, whereas it reduced the inhibitory effect of subthreshold BLA stimulation on vHipp-evoked $\mathrm{SP}$ in $\mathrm{mPFC}$ at short ISIs (*** $<0.01$ compared with the corresponded ISIs before PCP administration; repeated-measures ANOVA; Tukey's post hoc test).

recording the basal vHipp-evoked activity and vHipp baseline responses preceded by BLA stimulation at ISIs $130-10 \mathrm{~ms}$ for nine $\mathrm{mPFC}$ neurons (Figure 4), rats were administered PCP $(0.4 \mathrm{mg} / \mathrm{kg}$, i.v.), and the interaction between BLA/vHipp input was assessed at the same ISIs as before administration of the PCP. Analysis of these data revealed that PCP produced a significant effect on the interaction between BLA and vHipp inputs in mPFC (multiple-measures ANOVA; $\mathrm{F}(13,94)=10.208 ; p<0.001$ ). Further analyses showed that when no BLA stimulation was administered, there were no significant differences between before- and after-PCP treatment groups with respect to the firing probabilities evoked by single-pulse stimulation of the vHipp (Tukey's post hoc test, $p>0.05$ ), as well as when it was preceded by BLA stimulation at ISIs $130-40 \mathrm{~ms}$ (Figure 4). In contrast, PCP significantly reduced the inhibitory effect of subthreshold BLA stimulation on vHipp-evoked spike probability in the $\mathrm{mPFC}$ at short ISIs (ie, 10 and $20 \mathrm{~ms}$; Tukey's post hoc test; $p<0.01$; Figure 4 ), in a manner similar to that observed in MAM rats. This finding indicates that any differences between the control condition and the drug treatment condition could not be attributed to group differences in the firing probabilities evoked by single-pulse stimulation alone, and furthermore that NMDA blockade mimicked the effect of MAM treatment on paired-pulse inhibition of $\mathrm{MPFC}$ neurons.

\section{DISCUSSION}

The BLA is known to provide an important input to the $\mathrm{mPFC}$ that has a prominent role in the regulation of affective responses (Laviolette et al, 2005). The MPFC is also 
known to receive a converging context-related input from the vHipp (Maren and Quirk, 2004). However, the nature of the interaction between vHipp and BLA inputs in regulating mPFC neuron firing has not been examined previously. The present study shows the effects of coincident amygdala and hippocampal activation on MPFC neuron spike output and how it may be disrupted in this animal model of schizophrenia. In neurons receiving converging projections from BLA and vHipp, inputs from the BLA exert a powerful facilitatory action over hippocampal-evoked firing at longer ISIs (ie, 130-20 ms), whereas it decreased spike probability at shorter ISIs (ie, $20-0 \mathrm{~ms}$ ). In contrast, in MAM-treated rats subthreshold stimulation of the BLA increased evoked spike probability of vHipp stimulation in MPFC at all ISIs tested $(0-130 \mathrm{~ms})$; moreover, administration of PCP to control rats mimicked the effect found in the MAM-treated rats, in that it completely attenuated the inhibitory effect of BLA input on vHipp-evoked responses in MPFC at short ISIs. In addition, although stimulation of the vHipp did not significantly affect the response to BLA stimulation in control rats, in MAM rats vHipp stimulation decreased BLA-evoked firing at all ISIs shorter than $20 \mathrm{~ms}$ compared with normal rats.

\section{Modulatory Influence of BLA on vHipp-Evoked Firing in mPFC is Timing-Dependent}

The BLA was found to potently increase the ability of the vHipp to drive spike firing in the MPFC when the BLA input to the mPFC was stimulated at intervals $>20 \mathrm{~ms}$ before vHipp stimulation, but it decreased spike probabilities at short latencies. In contrast to the effects of BLA stimulation on vHipp-mPFC-evoked responses, there was no inhibitory period when examining the effects of vHipp stimulation on BLA-mPFC-evoked responses in control rats. Pyramidal neurons and $\mathrm{PV}$-immunoreactive GABAergic interneurons in different subregions of the $\mathrm{MPFC}$, including the prelimbic and infralimbic cortices, receive glutamatergic projections from the BLA (Gabbott et al, 2006; McDonald, 1991; Mcdonald, 1996; Shinonaga et al, 1994). Previous electrophysiological recordings reported that BLA inputs exerted both inhibition and excitation of MPFC neurons. Consistent with previous reports, these findings showed that the most prominent effect of BLA stimulation seems to be an evoked inhibition of mPFC neuron firing (Floresco and Tse, 2007; Pérez-Jaranay and Vives, 1991). The glutamatergic projections from the BLA that synapse onto PV-positive GABAergic interneurons provide one potential local feedforward inhibitory effect that can suppress firing of large groups of neurons (Gabbott et al, 1997, 2006). In a similar manner, afferent input of the hippocampus to the mPFC contacts both pyramidal neurons and inhibitory GABAergic interneurons (Gabbott et al, 2002). Similar effects have been observed using extracellular recordings of single-unit activity in the OMPFC in sodium pentobarbitalanesthetized rats, where an inhibitory influence of the BLA or CA1/subiculum on CA1/subiculum-induced or BLAinduced excitation was observed when the subsequent stimulation was given $20-40 \mathrm{~ms}$ before CA1/subiculum (or BLA) stimulation (Ishikawa and Nakamura, 2003). In contrast to the BLA input, we did not observe any inhibitory effect of the vHipp input on BLA-evoked firing in MPFC in controls. These findings suggest that the facilitation of hippocampal-evoked firing after stimulation of the BLA afferents preferentially activates feedforward excitatory circuits, provided by glutamatergic synapses on spines of the prefrontal pyramidal neurons (Gabbott et al, 2006). In contrast, the BLA projection seems to preferentially activate mPFC fast-spiking interneurons at shorter latencies compared with the pyramidal neurons.

\section{The Inhibitory Influence of the BLA on the vHipp-mPFC Pathway May be Produced Via NMDA Activation of Inhibitory Neurons}

A decrease in PV interneuron staining was observed throughout the mPFC and ventral subiculum of the hippocampus in MAM-treated rats (Lodge et al, 2009). This decrease appears specific for PV-containing interneurons, which is consistent with the reduction in local inhibitory GABAergic PV neuron staining that has been reported in postmortem studies in human schizophrenia patients (Lewis et al, 2005; Woo et al, 1998; Zhang and Reynolds, 2002). Thus, the reduction in PV interneuron functionality would likely affect the cortical pyramidal neuron output. We have shown that MAM-treated rats display a deficit in the ability to activate cortical and hippocampal assemblies during a latent inhibition paradigm (Lodge et al, 2009). These findings showed a deficit in gamma band activity in the MAM-treated rats in both the mPFC and vHipp, the same regions that show significant reductions in PV neuron staining (Lodge et al, 2009). Fastspiking PV interneurons have been shown to be necessary for the generation of gamma band activity (Bartos et al, 2002, 2007; Buzsáki et al, 1983; Lewis et al, 2011; Whittington et al, 1995), which is also disrupted in schizophrenia (Lewis et al, 2011, 2012). Our results showed that stimulation of the BLA induced an inhibition of vHippevoked firing in MPFC neurons at short latencies. These effects were reduced in MAM-treated rats in which the current studies show that activation of the BLA did not lead to a time-dependent inhibition over vHipp-evoked activity of PFC neurons. In contrast to the control rats, at ISIs shorter than $20 \mathrm{~ms}$ the vHipp input significantly attenuated the BLA response. These data suggest an altered excitatory/ inhibitory balance in the PFC of MAM-treated rats, in which BLA-evoked inhibition of the vHipp input is attenuated, but in which the vHipp now exerts a short-latency inhibition of the BLA-evoked mPFC drive. It is unclear why there would be a greater vHipp-BLA inhibitory control in MAM rats compared with controls. However, one possibility is that in the MAM rat the functional loss of PV interneurons leads to compensatory upregulation of other inhibitory process that are activated by vHipp inputs. Indeed, Volk and Lewis (2002) reported that in schizophrenia patients there is an upregulation of GABA A receptors on pyramidal neurons that is proposed to be because of the compensation for loss of inhibitory circuits. Such an upregulation could account for the emergence of the vHipp-mediated inhibition of BLAevoked responses.

In addition to being localized on some pyramidal neurons, NMDA channels in the PFC also reside on GABAergic interneurons (Gabbott et al, 2002, 2006). PCP induces schizophrenia-like symptoms in humans and 
frontal cortical dysfunction with corresponding symptoms of schizophrenia in non-human animals (Cohen et al, 1962; Moghaddam and Adams, 1998; Stefani and Moghaddam, 2002; Domino and Luby, 2012). In rodents, previous in vivo studies have shown that systemic administration of PCP elicits long-lasting activation of mPFC neurons, which occurs primarily via excitatory afferent inputs (Jodo et al, 2003; Suzuki et al, 2002). Injection of the NMDA channel blocker PCP attenuated the BLA-mediated inhibition of hippocampal-evoked firing, abolishing the timing-dependent inhibition that was observed under control conditions. This finding implies that NMDA channel activation on mPFC inhibitory interneurons might be responsible for the inhibitory actions of BLA input on MPFC neuron firing. Given that PV interneurons are known to be driven primarily by NMDA receptors, NMDA-mediated activation of PV interneurons should potently inhibit pyramidal cell output. This is consistent with studies showing that NMDA receptor blockade results in pyramidal neuron excitation via disinhibition (Homayoun and Moghaddam, 2007). These effects parallel those observed in MAM-treated rats during which BLA inputs to MPFC neurons did not inhibit vHipp-evoked firing at short latencies. PCP administration did not affect the facilitatory effect of the BLA input on vHipp-evoked spike probability as well as vHipp-driven spike firing at threshold level when stimulated alone, suggesting that PCP has a selective action on inhibitory circuits activated by BLA stimulation. This is consistent with our data showing that the loss of inhibitory function in the MAM rats is mimicked by blockade of NMDA function by PCP in control rats. It is likely that both effects observed at short latencies are due to an attenuation of BLA drive of interneurons, either owing to the decreased stimulationinduced activation of the population of PV-stained neurons (in the MAM rats) or blockade of excitatory NMDA drive on these neurons (in the PCP-treated rats).

\section{Functional Implications}

The results reported here have significant implications for cognitive processes in normal subjects as well as in pathological states. It is well known that the vHipp has a role in context-dependent processing (Maren and Quirk, 2004). The BLA is also known to provide an important input to the $\mathrm{mPFC}$ that has a prominent role in the regulation of affective responses (Laviolette et al, 2005). We demonstrated that a subpopulation of neurons of the $\mathrm{mPFC}$ receiving a functional input from the BLA can actively encode emotional learning (Laviolette et al, 2005). The neural networks incorporating the frontal, temporal lobes, and amygdala regulate fear memory, reward-associated learning, drug addiction, and anxiety disorders (Feltenstein and See, 2008; Fuchs et al, 2005; Maren, 2008; Orsini et al, 2011). The current study demonstrates that the BLA has an essential time-dependent facilitatory and inhibitory role in regulating vHipp-mPFC information flow, which may be significant in the gating of behavioral responses. Affective input arising in the BLA can attenuate vHipp drive when the context and affective inputs occur nearly simultaneously. In contrast, in cases where the affective stimulus occurs earlier, the system is apparently biased toward a greater response to a contextual stimulus arising in the vHipp.
Hippocampal hyperactivity is associated with psychosis in schizophrenia (Liddle et al, 1992; Silbersweig et al, 1995). We reported previously that the reduction in interneuron functionality in the vHipp and $\mathrm{mPFC}$ is correlated with a pathologically reduced gamma band response to a conditioned tone. The deficit in $\mathrm{mPFC}$ and ventral hippocampal oscillatory activity was associated with an impaired expression of latent inhibition in MAM-treated rats (Lodge et al, 2009). In addition to the vHipp, the amygdala may also have a role in schizophrenia (Reynolds, 1983). Indeed, we found that MAM-treated rats display a deficit in inhibitory effect of the BLA on vHipp-driven firing in mPFC when the activation of both is almost simultaneous. Furthermore, the present data suggest that a decreased NMDA channel activity may augment the excitability of mPFC neurons to vHipp input, rendering them more responsive to information arriving from the hippocampus, especially when this information arrives almost immediately after BLA input.

Collectively, these data suggest that inputs from the BLA and vHipp are essential components of cortical-subcortical neural networks that modulate information processing in the mPFC. Therefore, under normal conditions, a person may be focused on a task on the basis of its context, which is driven by hippocampal facilitation of information flow in the mPFC. However, in the presence of a threatening stimulus, the amygdala should be capable of producing an override of the context information at the level of the mPFC, facilitating those responses that are related to escape. This would enable the organism to respond to the threatening stimulus even if it is not congruent with the current context. However, in models of schizophrenia, the normal excitatory/inhibitory balance is altered, resulting in a preferential role for the vHipp in driving behavioral responses. It suggests that schizophrenia patients may respond to stimuli not on the basis of past experience or amygdala driving in $\mathrm{mPFC}$, but on the basis of their contextual valence. Dysfunction of these components would be expected to result in disorganized information processing, behavioral inflexibility, and cognitive dysfunction, as observed in schizophrenia.

\section{ACKNOWLEDGEMENTS}

This work was supported by United States Public Health Service Grant MH57440 (A A G). We thank Niki MacMurdo for technical assistance and Drs Pauline Belujon and Katy Gill for their helpful discussions.

\section{DISCLOSURE}

Dr Grace has received financial support from Johnson \& Johnson, Lundbeck, Pfizer, GSK, Puretech Ventures, Merck, Takeda, Dainippon Sumitomo, Otsuka, Lilly, Roche, and Asubio. Dr Esmaeili declares no conflict of interest.

\section{REFERENCES}

Allen P, Luigjes J, Howes OD, Egerton A, Hirao K, Valli I et al (2012). Transition to psychosis associated with prefrontal and subcortical dysfunction in ultra high-risk individuals. Schizophr Bull 38: 1268-1276. 
Bacon SJ, Headlam AJ, Gabbott PL, Smith AD (1996). Amygdala input to medial prefrontal cortex (mPFC) in the rat: a light and electron microscope study. Brain Res 720: 211-219.

Bartos M, Vida I, Frotscher M, Meyer A, Monyer H, Geiger JRP et al (2002). Fast synaptic inhibition promotes synchronized gamma oscillations in hippocampal interneuron networks. Proc Natl Acad Sci USA 99: 13222-13227.

Bartos M, Vida I, Jonas P (2007). Synaptic mechanisms of synchronized gamma oscillations in inhibitory interneuron networks. Nat Rev Neurosci 8: 45-56.

Behrens MM, Ali SS, Dao DN, Lucero J, Shekhtman G, Quick KL et al (2007). Ketamine-induced loss of phenotype of fastspiking interneurons is mediated by NADPH-oxidase. Science 318: 1645-1647.

Benes FM (2010). Amygdalocortical circuitry in schizophrenia: from circuits to molecules. Neuropsychopharmacology 35: 239-257.

Berretta S, Lange N, Bhattacharyya S, Sebro R, Garces J, Benes FM (2004). Long-term effects of amygdala GABA receptor blockade on specific subpopulations of hippocampal interneurons. Hippocampus 14: 876-894.

Bitanihirwe BKY, Lim MP, Kelley JF, Kaneko T, Woo TUW (2009). Glutamatergic deficits and parvalbumin-containing inhibitory neurons in the prefrontal cortex in schizophrenia. $B M C$ Psychiatry 9: 71.

Buzsáki G, Leung LW, Vanderwolf CH (1983). Cellular bases of hippocampal EEG in the behaving rat. Brain Res 287: 139-171.

Carr DB, Sesack SR (1996). Hippocampal afferents to the rat prefrontal cortex: synaptic targets and relation to dopamine terminals. J Comp Neurol 369: 1-15.

Cohen BD, Rosenbaum G, Luby ED, Gottlieb JS (1962). Comparison of phencyclidine hydrochloride (Sernyl) with other drugs. Simulation of schizophrenic performance with phencyclidine hydrochloride (Sernyl), lysergic acid diethylamide (LSD-25), and amobarbital (Amytal) sodium; II. Symbolic and sequential think. Arch Gen Psychiatry 6: 395-401.

Corcoran KA, Desmond TJ, Frey KA, Maren S (2005). Hippocampal inactivation disrupts the acquisition and contextual encoding of fear extinction. J Neurosci 25: 8978-8987.

Coyle JT (1996). The glutamatergic dysfunction hypothesis for schizophrenia. Harv Rev Psychiatry 3: 241-253.

Cunningham MO, Hunt J, Middleton S, LeBeau FEN, Gillies MJ, Gillies MG et al (2006). Region-specific reduction in entorhinal gamma oscillations and parvalbumin-immunoreactive neurons in animal models of psychiatric illness. J Neurosci 26: 2767-2776.

Domino EF, Luby ED (2012). Phencyclidine/schizophrenia: one view toward the past, the other to the future. Schizophr Bull 38: 914-919.

Feltenstein MW, See RE (2008). The neurocircuitry of addiction: an overview. Br J Pharmacol 154: 261-274.

Flagstad P, Mørk A, Glenth $\varnothing$ j BY, Beek J van, Michael-Titus AT, Didriksen M (2004). Disruption of neurogenesis on gestational day 17 in the rat causes behavioral changes relevant to positive and negative schizophrenia symptoms and alters amphetamineinduced dopamine release in nucleus accumbens. Neuropsychopharmacology 29: 2052-2064.

Floresco SB, Grace AA (2003). Gating of hippocampal-evoked activity in prefrontal cortical neurons by inputs from the mediodorsal thalamus and ventral tegmental area. J Neurosci 23: 3930-3943.

Floresco SB, Tse MT (2007). Dopaminergic regulation of inhibitory and excitatory transmission in the basolateral amygdalaprefrontal cortical pathway. J Neurosci 27: 2045-2057.

Fuchs RA, Evans KA, Ledford CC, Parker MP, Case JM, Mehta RH et al (2005). The role of the dorsomedial prefrontal cortex, basolateral amygdala, and dorsal hippocampus in contextual reinstatement of cocaine seeking in rats. Neuropsychopharmacology 30: 296-309.
Gabbott P, Headlam A, Busby S (2002). Morphological evidence that CA1 hippocampal afferents monosynaptically innervate PVcontaining neurons and NADPH-diaphorase reactive cells in the medial prefrontal cortex (Areas 25/32) of the rat. Brain Res 946: 314-322.

Gabbott PL, Dickie BG, Vaid RR, Headlam AJ, Bacon SJ (1997). Local-circuit neurones in the medial prefrontal cortex (areas 25, 32 and 24b) in the rat: morphology and quantitative distribution. J Comp Neurol 377: 465-499.

Gabbott PLA (2003). Radial organisation of neurons and dendrites in human cortical areas 25, 32, and 32'. Brain Res 992: 298-304.

Gabbott PLA, Warner TA, Busby SJ (2006). Amygdala input monosynaptically innervates parvalbumin immunoreactive local circuit neurons in rat medial prefrontal cortex. Neuroscience 139: 1039-1048.

Garcia R, Vouimba RM, Baudry M, Thompson RF (1999). The amygdala modulates prefrontal cortex activity relative to conditioned fear. Nature 402: 294-296.

Gigg J, Tan AM, Finch DM (1994). Glutamatergic hippocampal formation projections to prefrontal cortex in the rat are regulated by GABAergic inhibition and show convergence with glutamatergic projections from the limbic thalamus. Hippocampus 4: 189-198.

Goldberg TE, Weinberger DR (1988). Probing prefrontal function in schizophrenia with neuropsychological paradigms. Schizophr Bull 14: 179-183.

Goto Y, Grace AA (2006). Alterations in medial prefrontal cortical activity and plasticity in rats with disruption of cortical development. Biol Psychiatry 60: 1259-1267.

Gourevitch R, Rocher C, Pen G Le, Krebs M-O, Jay TM (2004). Working memory deficits in adult rats after prenatal disruption of neurogenesis. Behav Pharmacol 15: 287-292.

Grace AA, Moore H (1998). Regulation of information flow in the nucleus accumbens: a model for the pathophysiology of schizophrenia. In: Lenzenweger MF, Dworkin RH (eds). Origins and development of schizophrenia: advances in experimental psychopathology. American Psychological Association: Washington, DC, pp 123-157.

Homayoun H, Moghaddam B (2007). NMDA receptor hypofunction produces opposite effects on prefrontal cortex interneurons and pyramidal neurons. J Neurosci 27: 11496-11500.

Ishikawa A, Nakamura S (2003). Convergence and interaction of hippocampal and amygdalar projections within the prefrontal cortex in the rat. J Neurosci 23: 9987-9995.

Javitt DC, Zukin SR (1991). Recent advances in the phencyclidine model of schizophrenia. Am J Psychiatry 148: 1301-1308.

Jay TM, Glowinski J, Thierry AM (1995). Inhibition of hippocampoprefrontal cortex excitatory responses by the mesocortical DA system. Neuroreport 6: 1845-1848.

Ji J, Maren S (2007). Hippocampal involvement in contextual modulation of fear extinction. Hippocampus 17: 749-758.

Jodo E, Suzuki Y, Takeuchi S, Niwa S, Kayama Y (2003). Different effects of phencyclidine and methamphetamine on firing activity of medial prefrontal cortex neurons in freely moving rats. Brain Res 962: 226-231.

Laviolette SR, Lipski WJ, Grace AA (2005). A subpopulation of neurons in the medial prefrontal cortex encodes emotional learning with burst and frequency codes through a dopamine D4 receptor-dependent basolateral amygdala input. J Neurosci 25: 6066-6075.

Lewis DA (2012). Cortical circuit dysfunction and cognitive deficits in schizophrenia-implications for preemptive interventions. Eur J Neurosci 35: 1871-1878.

Lewis DA, Curley AA, Glausier JR, Volk DW (2012). Cortical parvalbumin interneurons and cognitive dysfunction in schizophrenia. Trends Neurosci 35: 57-67. 
Lewis DA, Fish KN, Arion D, Gonzalez-Burgos G (2011). Perisomatic inhibition and cortical circuit dysfunction in schizophrenia. Curr Opin Neurobiol 21: 866-872.

Lewis DA, Hashimoto T (2007). Deciphering the disease process of schizophrenia: the contribution of cortical GABA neurons. Int Rev Neurobiol 78: 109-131.

Lewis DA, Hashimoto T, Volk DW (2005). Cortical inhibitory neurons and schizophrenia. Na Rev Neurosci 6: 312-324.

Lewis DA, Moghaddam B (2006). Cognitive dysfunction in schizophrenia: convergence of gamma-aminobutyric acid and glutamate alterations. Arch Neurol 63: 1372-1376.

Liddle PF, Friston KJ, Frith CD, Hirsch SR, Jones T, Frackowiak RS (1992). Patterns of cerebral blood flow in schizophrenia. $\mathrm{Br} J$ Psychiatry 160: 179-186.

Lodge DJ, Behrens MM, Grace AA (2009). A loss of parvalbumincontaining interneurons is associated with diminished oscillatory activity in an animal model of schizophrenia. J Neurosci 29: 2344-2354.

Maren S (2008). Pavlovian fear conditioning as a behavioral assay for hippocampus and amygdala function: cautions and caveats. Eur J Neurosci 28: 1661-1666.

Maren S, Quirk GJ (2004). Neuronal signalling of fear memory. Nat Revi Neurosci 5: 844-852.

McDonald AJ (1991). Organization of amygdaloid projections to the prefrontal cortex and associated striatum in the rat. Neuroscience 44: 1-14.

Mcdonald AJ (1996). Glutamate and aspartate immunoreactive neurons of the rat basolateral amygdala: colocalization of excitatory amino acids and projections to the limbic circuit. $J$ Comp Neurol 365: 367-379.

Milad MR, Quirk GJ (2002). Neurons in medial prefrontal cortex signal memory for fear extinction. Nature 420: 70-74.

Milad MR, Vidal-Gonzalez I, Quirk GJ (2004). Electrical stimulation of medial prefrontal cortex reduces conditioned fear in a temporally specific manner. Behav Neurosci 118: 389-394.

Moghaddam B, Adams BW (1998). Reversal of phencyclidine effects by a group II metabotropic glutamate receptor agonist in rats. Science 281: 1349-1352.

Moore H, Jentsch JD, Ghajarnia M, Geyer MA, Grace AA (2006). A neurobehavioral systems analysis of adult rats exposed to methylazoxymethanol acetate on E17: implications for the neuropathology of schizophrenia. Biol Psychiatry 60: 253-264.

Orsini CA, Kim JH, Knapska E, Maren S (2011). Hippocampal and prefrontal projections to the basal amygdala mediate contextual regulation of fear after extinction. J Neurosci 31: 17269-17277.

Paxinos G, Watson C (1986). The rat brain in stereotaxic coordinates. Academic: Sydney.

Penschuck S, Flagstad P, Didriksen M, Leist M, Michael-Titus AT (2006). Decrease in parvalbumin-expressing neurons in the hippocampus and increased phencyclidine-induced locomotor activity in the rat methylazoxymethanol (MAM) model of schizophrenia. Eur J Neurosci 23: 279-284.

Pérez-Jaranay JM, Vives F (1991). Electrophysiological study of the response of medial prefrontal cortex neurons to stimulation of the basolateral nucleus of the amygdala in the rat. Brain Res 564: 97-101.

Phillips RG, LeDoux JE (1992). Differential contribution of amygdala and hippocampus to cued and contextual fear conditioning. Behav Neurosci 106: 274-285.

Reynolds GP (1983). Increased concentrations and lateral asymmetry of amygdala dopamine in schizophrenia. Nature 305: 527-529.

Rosenkranz JA, Grace AA (2002). Dopamine-mediated modulation of odour-evoked amygdala potentials during pavlovian conditioning. Nature 417: 282-287.

Santana N, Troyano-Rodriguez E, Mengod G, Celada P, Artigas F (2011). Activation of thalamocortical networks by the N-methylD-aspartate receptor antagonist phencyclidine: reversal by clozapine. Biol Psychiatry 69: 918-927.

Selden NR, Everitt BJ, Jarrard LE, Robbins TW (1991). Complementary roles for the amygdala and hippocampus in aversive conditioning to explicit and contextual cues. Neuroscience 42: 335-350.

Shinonaga Y, Takada M, Mizuno N (1994). Topographic organization of collateral projections from the basolateral amygdaloid nucleus to both the prefrontal cortex and nucleus accumbens in the rat. Neuroscience 58: 389-397.

Silbersweig DA, Stern E, Frith C, Cahill C, Holmes A, Grootoonk S et al (1995). A functional neuroanatomy of hallucinations in schizophrenia. Nature 378: 176-179.

Stefani MR, Moghaddam B (2002). Effects of repeated treatment with amphetamine or phencyclidine on working memory in the rat. Behav Brain Res 134: 267-274.

Suzuki Y, Jodo E, Takeuchi S, Niwa S, Kayama Y (2002). Acute administration of phencyclidine induces tonic activation of medial prefrontal cortex neurons in freely moving rats. Neuroscience 114: 769-779.

Volk DW, Lewis DA (2002). Impaired prefrontal inhibition in schizophrenia: relevance for cognitive dysfunction. Physiol Behav 77: 501-505.

Whittington MA, Traub RD, Jefferys JG (1995). Synchronized oscillations in interneuron networks driven by metabotropic glutamate receptor activation. Nature 373: 612-615.

Wolf RC, Vasic N, Sambataro F, Höse A, Frasch K, Schmid M et al (2009). Temporally anticorrelated brain networks during working memory performance reveal aberrant prefrontal and hippocampal connectivity in patients with schizophrenia. Prog Neuropsychopharmacol Biol Psychiatry 33: 1464-1473.

Woo TU, Whitehead RE, Melchitzky DS, Lewis DA (1998). A subclass of prefrontal gamma-aminobutyric acid axon terminals are selectively altered in schizophrenia. Proc Natl Acad Sci USA 95: 5341-5346.

Zhang ZJ, Reynolds GP (2002). A selective decrease in the relative density of parvalbumin-immunoreactive neurons in the hippocampus in schizophrenia. Schizophr Res 55: 1-10.

Zhou Y, Shu N, Liu Y, Song M, Hao Y, Liu H et al (2008). Altered resting-state functional connectivity and anatomical connectivity of hippocampus in schizophrenia. Schizophr Res 100: 120-132. 